Note

\title{
SPANNING TREES WHOSE STEMS HAVE A BOUNDED NUMBER OF BRANCH VERTICES
}

\author{
ZHENG YAN \\ Institute of Applied Mathematics \\ Yangtze University, Jingzhou, Hubei, China \\ e-mail: yanzhenghubei@163.com
}

\begin{abstract}
Let $T$ be a tree, a vertex of degree one and a vertex of degree at least three is called a leaf and a branch vertex, respectively. The set of leaves of $T$ is denoted by Leaf $(T)$. The subtree $T-\operatorname{Leaf}(T)$ of $T$ is called the stem of $T$ and denoted by $\operatorname{Stem}(T)$. In this paper, we give two sufficient conditions for a connected graph to have a spanning tree whose stem has a bounded number of branch vertices, and these conditions are best possible.
\end{abstract}

Keywords: spanning tree, stem, branch vertex.

2010 Mathematics Subject Classification: 05C70.

\section{REFERENCES}

[1] E. Flandrin, T. Kaiser, R. Kužel, H. Li and Z. Ryjăćek, Neighborhood unions and extremal spanning trees, Discrete Math. 308 (2008) 2343-2350. doi:10.1016/j.disc.2007.04.071

[2] L. Gargano and M. Hammar, There are spanning spiders in dense graphs (and we know how to find them), Lect. Notes Comput. Sci. 2719 (2003) 802-816. doi:10.1007/3-540-45061-0_63

[3] L. Gargano, M. Hammar, P. Hell, L. Stacho and U. Vaccaro, Spanning spiders and light-splitting switchs, Discrete Math. 285 (2004) 83-95.

doi:10.1016/j.disc.2004.04.005

[4] L. Gargano, P. Hell, L. Stacho and U. Vaccaro, Spanning trees with bounded number of branch vertices, Lect. Notes Comput. Sci. 2380 (2002) 355-365. doi:10.1007/3-540-45465-9_31

[5] M. Kano, M. Tsugaki and G. Yan, Spanning trees whose stems have bounded degrees, preprint. 
[6] M. Kano and Z. Yan, Spanning trees whose stems have at most $k$ leaves, Ars Combin. CXIVII (2014) 417-424.

[7] A. Kyaw, Spanning trees with at most 3 leaves in $K_{1,4}$-free graphs, Discrete Math. 309 (2009) 6146-6148. doi:10.1016/j.disc.2009.04.023

[8] H. Matsuda, K. Ozeki and T. Yamashita, Spanning trees with a bounded number of branch vertices in a claw-free graph, Graphs Combin. 30 (2014) 429-437. doi:10.1007/s00373-012-1277-5

[9] M. Tsugaki and Y. Zhang, Spanning trees whose stems have a few leaves, Ars Combin. CXIV (2014) 245-256.

Received 1 June 2015

Revised 29 October 2015

Accepted 19 November 2015 